\title{
EINIGE ÜBERLEGUNGEN ZU DER ZWEITEN GRUPPE NAVIGANTES IN AUGUSTINS DIALOG DE BEATA VITA
}

\begin{abstract}
Summary: At the beginning of the series of Augustine's earliest extant literary works are the three philosophical dialogues Contra Academicos, De beata vita and De ordine, which were composed in the autumn of 386 during Augustine's otium philosophandi in Cassiciacum. In the introductions of all three works, the marine metaphors are widely used. The author compares human life with a stormy sea and sees as the only salvation the port of philosophy. In beata v. 1.2 Augutin compares the people to whom philosophy can accommodate with navigantes, which he groups in tria genera. Although the people who belong to the respective group are described in detail, the author does not mention names. This arouses research interest and justifies the attempt to propose a representing person for each group.

The present work sets itself the goal by parallel reading of beata $v .1 .2$, with some passages from Cicero's Epistulae, Tusculanae disputationes and De officiis to make a new proposal. And this in addition to the currently existing assumption that Romanianus, to whom the dialogue Contra Academicos is dedicated, should be considered as a representative of the second group of seafarers. However, the author of the present work now ventures a completely new approach, in which Cicero can be accepted as a representative of this group.
\end{abstract}

Keywords: Augustine, Cicero, Dialogues of Cassiciacum, philosophy, mariners

Im Herbst 386, nach Aufgabe seines Postens als Rhetorikprofessor am Mailänder Hof zieht sich der junge Augustin auf das Landgut seines Freundes Verecundus ${ }^{1}$ in Cassiciacum $^{2}$ zurück, um sich zusammen mit einem Kreis von Schülern und Verwandten

${ }^{1}$ Vgl. ord. 1. 2. 5: ad villam familiarissimi nostri Verecundi.

${ }^{2} \mathrm{Im}$ corpus Augustinianum ist die Bezeichnung rus Cassiciacum nur in conf. 9. $3.5 \mathrm{zu}$ finden: pro rure illo eius (scil. Verecundi) Cassiciaco. Die genaue Lage von Cassiciacum ist bis in die neuere Zeit Gegenstand einer Kontroverse. Augustins Angaben in den Dialogen und in conf., topographische, archäologische und toponymische Erwähnungen sprechen jedoch klar für Cassago Brianza (ca. 40 km nordwestlich von Mailand) und gegen Casciago bei Varese (ca. $55 \mathrm{~km}$ nordwestlich von Mailand). Dazu vgl. die ausführliche Literatur bei TRELENBERG, J.: Augustins Schrift De ordine. Einführung, Kommentar, Ergebnisse. Tübingen 2009, 68, mit Anm. 78. 
dem otium philosophandi ${ }^{3}$ zu widmen. Seine Studien in dieser Zeit charakterisiert der Autor als Wahrheitssuche. ${ }^{4}$ Der amor philosophiae, der laut Augustins eigener Aussage in beata v. 1.4 durch die Beschäftigung mit Ciceros protreptischer Schrift Hortensius $^{5}$ entstanden ist, wird die treibende Kraft in seinem Leben. Die drei - nach ihrem Entstehungsort genannten - Cassiciacum-Dialoge Contra Academicos, De beata vita und De ordine $e^{6}$ entstehen aus der Bearbeitung der von einem notarius ${ }^{7}$ gemachten Aufzeichnungen der Gespräche, die Augustin mit seinen Begleitern führte.

In den Proömien aller drei Dialoge wird die Meeresmetaphorik breit verwendet. In beata v. $1.1 \mathrm{im}$ Anschluss an die einleitende Anrede an Theodorus vergleicht Augustin die Welt mit einem stürmischen Meer, in welches die Menschen hineingeworfen sind. Die einzige Rettung ist der portus philosophiae. ${ }^{8}$ Das Vorbild findet sich bei Cicero (vgl. Tusc. 5. 5: Cuius (scil. philosophiae) in sinum cum a primis temporibus aetatis nostra voluntas studiumque nos compulisset, his gravissimis casibus in eundem portum, ex quo eramus egressi, magna iactati tempestate confugimus; fam. 7. 30. 2: Sunt enim innumerabilia...; quae quidem ego non ferrem, nisi me in philosophiae portum contulissem...).

Die Menschen, denen die Philosophie ${ }^{9}$ Aufnahme gewähren kann, teilt Augustin in drei Arten Seefahrer ein, jede von ihnen mit unterschiedlichem Zugang zum Hafen der Philosophie. Das Vorbild für diese Einteilung ist Senecas Referat von Epikur, der die Menschen, die zu Wahrheit kommen, in drei Gruppen ordnet, in epist. 52. $3-4^{10}$. Laut der Einteilung von Epikur gehören zu der ersten Gruppe Menschen, die ohne Hilfe zur Wahrheit kommen. ${ }^{11}$ Die Menschen, die in der zweiten Gruppe eingeteilt sind, brauchen fremde Hilfe. ${ }^{12}$ Die Menschen in der dritten Gruppe sind jene, die einen Helfer und sogar einen Antreiber brauchen. ${ }^{13}$ Epikur gibt je ein Beispiel für Menschen, die zu der zweiten und zu der dritten Gruppe gehören: Metrodoros bzw. Hermarchos. Die erste Gruppe lässt Epikur ohne konkreten Repräsentanten.

\footnotetext{
${ }^{3}$ Vgl. Acad. 2. 2. 4.

${ }^{4}$ Vgl. Acad. 2. 2. 4; ord. 1. 3. 6; sol. 2. 17. 31; ep. 1. 3.

${ }^{5} \mathrm{Zu}$ Ciceros protreptischem Dialog Hortensius und zu Augustins eigenem Lektüreerlebnis vgl. SCHLAPBACH, K.: Augustin Contra Academicos Buch I. Einleitung und Kommentar. Berlin 2003, 14-16.

${ }^{6}$ Alle Zitate aus De beata vita, Contra Academicos und De ordine folgen der Ausgabe von W. M. GREEN, CCSL 29, Turnhout 1970.

${ }^{7}$ Zur Stenographie vgl. Acad. 1. 5. 15; 2. 7. 17; 2. 13. 29; 3. 7. 15; 3. 20. 44; beata v. 2. 15; 3, 18; ord. 1. 10. 29 f.; 1. 11.33; 2. 7. 21.

${ }^{8}$ Das Bild des Hafens der Philosophie (bzw. der Weisheit) ist ein Topos. Vgl. z. B. Acad. 1. 1. 1; 2. 1. 1. Weitere Bilder für die Philosophie als Zufluchtsort sind der "Schoss“ vgl. Acad. 2. 3. 7; beata v. 1. 5 und die „Wohnung“"vgl. ord. 1.3.6.

${ }_{9}$ Zur Tradition der Personifikation der Philosophie vgl. SCHLAPBACH (Anm. 5) 55, [72].

${ }^{10}$ Dazu vgl. Valenzano, M.: La dedica del "De beata vita" di S. Agostino. Civiltà classica e cristiana 3 (1982) 337-352, hier 342-344.

${ }^{11}$ Sen. epist. 52. 3: ... ad veritatem sine ullius adiutorio exisse, fecisse sibi ipsos viam.

${ }^{12}$ Ibid.: quosdam indigere ope aliena, non ituros si nemo praecesserit, sed bene secuturos.

${ }^{13}$ Sen. epist. 52. 4: Praeter haec adhuc invenies genus aliud hominum ne ipsum quidem fastidiendum eorum qui cogi ad rectum compellique possunt, quibus non duce tantum opus sit sed adiutore et, ut ita dicam, coactore.
} 
Die erste Art von Menschen, die Augustin in beata v. 1. 2 beschreibt, sind jene, die sich noch jung (Z. 17: ubi aetas compos rationis adsumpserit), mühelos, mit leichtem Ruderschlag aus der Küstennähe in den Hafen flüchten (Z. 17 f.: parvo impetu pulsuque remorum de proximo fugiunt). Dort richten sie irgendein eigenes Werk auf (Z. 20 f.: sui alicuius operis erigunt), welches für die anderen ein Leuchtzeichen (Z. 20: lucidissimum signum) sein soll.

Die zweite Art von Menschen, die beschrieben sind, sind diese, die getäuscht von der trügerischen Oberfläche des Meeres, ${ }^{14}$ es wagen hinauszufahren, fern ihrer Heimat ${ }^{15}$ umherzuschweifen (Z. 21-23: qui fallacissima facie maris decepti elegerunt in medium progredi longeque a sua patria peregrinari audent), und dabei wird sie oft vergessen (Z. 23 f.: et eius (scil. patriae) obliviscuntur). Diese Leute, vorangetrieben von einem scheinbar günstigen Rückenwind (Z. 24 f.: a puppi ventus, quem prosperum putant), gelangen in tiefstes Elend (Z. 25 f.: penetrant in altissima miseriarum). Die Schwierigkeit ihrer Lage können sie überhaupt nicht verstehen, weil sie hochgestimmt und froh sind (Z. 26: elati atque gaudentes), da der trügerische Glanz von Vergnügungen und Ehren sie umschmeichelt (Z. 26-28: quod eis usque quaque fallacissima serenitas voluptatum honorumque blanditur $\left.{ }^{16}\right)$. Das Einzige, was diesen Menschen zu wünschen ist, ist widriges Wetter und sogar tobender Sturm und Gegenwind (Z. 29 f.: inprospera et ... saeviens omnino tempestas contrariaque flans ventus), um sie zu echten Freuden (Z. 31: certa et solida gaudia) hinzuführen, wenn sie auch weinen und klagen (Z. 31 flentes gementesque). Das sind Menschen, die durch tränenreiche Tragödien ihrer Schicksale (Z. 34 f.: lacrimabiles tragoediae fortunarum suarum) oder durch beklemmende Schwierigkeiten bei ihren eitlen Geschäften (Z. 35: inanium negotiorum anxiae difficultates) auf die Bücher gelehrter und weiser Männer gestossen werden (Z. 36 f.: in libros doctorum sapientissimorumque hominum truserint), da ihnen kaum etwas anderes mehr zu tun bleibt (quasi nihil aliud habentes, quod agant). Diese Menschen wachen dann genau im Hafen auf (Z. 37 f.: in ipso ... portu evigilant $^{17}$ ) und keine Verheißungen des Meeres können sie mehr hinauslocken (Z. 38 f.: nulla maris illius promissa nimium falso ridentis excludant).

Die dritte Art der Menschen, welche die Philosophie aufnehmen kann, sind jene, die trotz langer und vieler Verwirrungen (Z. 40: iam diu multumque iactati) dennoch gewisse Wegzeichen erblicken (Z. 41: quaedam signa respiciunt) und sich auf hohem Meer (Z. 42: in ipsis fluctibus) an ihre teure Heimat erinnern (Z. 41 f.: suae dulcissimae patriae ... recordantur ${ }^{18}$ ). Sie kehren dann entweder ohne Umweg und weitere Verzögerungen zu ihr zurück (Z. 42 f.: recto cursu in nullo falsi et nihil morati eam (scil. patriam) repetunt) oder sie kommen bei schlechter Sicht vom Kurs ab

\footnotetext{
${ }^{14}$ Vgl. Lucr. 5. 1005 und Aug. ep. 15. 2.

${ }^{15}$ Mit dem Bild des Hafens ist das Motiv der Rückkehr in die Heimat verbunden. Vgl. z. B. Acad. 3. 20. 42 .

${ }^{16}$ Blandiri-zum Genuss einladen, verlocken, wohltun (vgl. SLA s.v. blandior, 2. 2) - wird meist mit negativer Konnotation verwendet.

${ }_{17}$ Zur Metapher des Aufwachens vgl. SCHLAPBACH (Anm. 5) 52 f. [67].

${ }^{18} \mathrm{Im}$ Hintergrund des Gedankens steht die platonische Anamnesis-Lehre.
} 
(Z. 44: inter nubila deviantes), orientieren sich an sinkenden Gestirnen (Z. 44: mergentia contuentes sidera), gefangen von mancher Verlockung (Z. 45: nonnulis inlecebris capti) lassen sie die Zeiten guter Fahrt verstreichen (Z. 45 f.: bonae navigationis tempora differentes errant) und geraten oft in Gefahr (Z. 46: saepe etiam periclitantur). Häufig werden sie auch durch einen Sturm, der all ihren Anstrengungen spottet (Z. 47 f.: conatibus eorum adversa tempestas), in das heiß ersehnte ruhevolle Leben (Z. 48 f.: in optatissimama vitam quietamque) gebracht.

Die Ausführlichkeit der Beschreibung der Menschen, die zu der zweiten Gruppe Seefahrer gehören, kontrastiert mit der Kürze der Beschreibung der navigantes der ersten und der dritten Gruppe: erste Gruppe: 1. 2. 16-21; zweite Gruppe: 1. 2. 21-39; dritte Gruppe: 1. 2. 39-49. Dieses Ungleichgewicht im Text fällt gleich ins Auge. Natürlich stellt sich der Leser die Frage: Warum hat Augustin den Text an dieser Stelle asymmetrisch konstruiert? Zufällig soll es nicht sein. Die weitere Arbeit versucht, dieser Frage eine mögliche Antwort anzubieten.

Bei einem Vergleich zwischen dem oben erwähnten 52. Brief von Seneca und Augustins beata $v$. fällt einem der Unterschied gleich auf, dass, während Seneca Beispiele gibt, die laut Epikur die zweite und die dritte Gruppe der Wahrheit-Suchenden repräsentieren, beschreibt Augustin die Menschen der einzelnen Gruppen zwar detailliert, gibt aber keine Beispiele dafür, wer zu den jeweiligen Gruppen gehört. Gut geschult in der antiken Tradition, welcher Augustin zur Zeit der Entstehung der Cassiciacum-Dialoge folgt, könnte es ihm nicht unbekannt gewesen sein, dass in der römischen Literatur gerne menschliche Vorbilder verwendet werden. An dieser Stelle soll die zweite Frage, deren Antwort die vorliegende Arbeit sucht, gestellt werden: Warum nennt Augustin keine Beispiele für Repräsentanten der jeweiligen Gruppe von Seefahrern?

Die modernen Interpreten versuchen anhand Augustins Beschreibungen der drei Arten von Menschen in jeder Gruppe eine Person zu erkennen. Doignon ${ }^{19}$ sieht in der ersten Gruppierung den Neuplatoniker Theodorus (den Adressaten des Dialogs De beata vita) gemeint, in der zweiten Augustins Gönner Romanianus (den Adressaten von Contra Academicos), in der dritten Augustin selbst. Molina ${ }^{20}$ sieht dagegen in der dritten Gruppierung „el tipo de la personalidad agustiniana“. Die vorliegende Arbeit stimmt den beiden oberen Zuordnungen $\mathrm{zu}$, versucht aber einen neuen Vorschlag für den Repräsentanten der zweiten Gruppe anzubieten.

Bei einem parallelen Lesen von Augustins Beschreibung der zweiten Art Menschen, die sich durch schwere private Schicksalsschläge und Unglück in ihrer Karriere der Philosophie zugewandt haben, mit einigen Stellen von Ciceros Schriften, welche in der Periode 46-44 v. Chr. entstanden sind, als Cicero nicht mehr am politischen Leben in Rom teilnehmen durfte, können viele Übereinstimmungen zwischen Augustins Beschreibung der zweiten Gruppe Seefahrer und Ciceros Beschreibung des

\footnotetext{
${ }^{19}$ Dolgnon, J.: La Vie Heureuse. Paris 1986, $133 \mathrm{f}$.

${ }^{20}$ MolinA, M. A.: Felicidad y sabiduría: Agustín en noviembre del 386. In Augustinus. Revista Trimestral Publicada Por Los Padres Agustinos Recoletos 18 (1973) 355-372, hier 361.
} 
eigenen Lebens in Epistulae, ${ }^{21}$ Tusculanae disputationes ${ }^{22}$ und De officiis ${ }^{23}$ festgestellt werden.

In einem in Rom geschriebenen Brief vom sechsten August 46 v. Chr. schildert Cicero seinem Freund Lucius Papirius Paetus seinen Tagesablauf. Nach der morgendlichen salutatio widmet er sich den Schriften - entweder er liest oder er schreibt: fam. 9. 20. 2: ubi salutatio defluxit, litteris me involvo: aut scribo aut lego... Diese Stelle stimmt mit beata v. 1. 2. 24-26 überein: quasi nihil aliud habentes, quod agant, in libros doctorum sapientissimorumque hominum truserint.

In off. 3. 1. 2 beschreibt Cicero sein otium bzw. seine philosophischen Studien als das Einzige, was ihm zu tun übrig blieb, nachdem er nicht mehr im Senat und am Gericht tätig gewesen war: nostrum autem otium negotii inopia, non requiescendi studio constitutum est. Extincto enim senatu deletisque iudiciis quid est, quod dignum nobis aut in curia aut in foro agere possimus?

Gemäß off. 2. 1. 4 kommt Cicero zur Philosophie, weil sein Geist nicht ohne Beschäftigung sein konnte: Nihil agere autem cum animus non posset..., d. h. die Philosophie war für ihn, nach dem Verlust des politischen Einflusses, die einzig mögliche Tätigkeit. Weiter klagt er darüber, dass ihm während seiner politischen Arbeit keine Zeit zum Schreiben geblieben ist: posteaquam honoribus inservire coepi meque totum rei publicae tradidi, tantum erat philosophiae loci, quantum superfuerat amicorum et rei publicae tempori. Id autem omne consumebatur in legendo, scribendi otium non erat.

Im Zeitraum 46-44 v. Chr. erlebt Cicero schwere Schicksalsschläge. In einem Brief vom März 45 v. Chr. tröstet Servius Sulpicius Rufus den um seine im Februar verstorbene Lieblingstochter Tullia trauernden Cicero. In seiner consulatio erwähnt er alles, was Cicero bereits verloren hat: Cogita, quemadmodum adhuc fortuna nobiscum egerit: ea nobis erepta esse, quae hominibus non minus quam liberi cara esse debent, patriam, honestatem, dignitatem, honores omnes.

In Tusc. 5.3 spricht Cicero selbst darüber, dass er vom Schicksal auf eine scharfe Probe gestellt wurde: Equidem eos casus, in quibus me fortuna vehementer exercuit... Damit sollen einerseits der Tod Tullias, andererseits die Schwierigkeiten, die er als Politiker und Jurist nach dem Tod Caesars bekommen hat, gemeint sein. ${ }^{24}$ An dieser Stelle kann wieder eine Parallele zu der zweiten Gruppe von Seefahrer, welche Augustin beschreibt, gezogen werden. Das sind Leute, die laut beata v. 1. 2 einerseits tränenreiche Tragödien ihrer Lebensschicksale oder beklemmende Schwierigkeiten bei ihren eitlen Geschäften erlebt haben. In jenem Lebensabschnitt, in dem Cicero sich der Philosophie zuwandte, befand er sich in einer privaten Tragödie we-

${ }^{21}$ Alle Zitate aus Epistulae folgen: M. Tullius Cicero, Epistulae ad familiares. Hrsg. von D. R. SHACKLETON BAILEY. Stuttgart 1988.

${ }^{22}$ Alle Zitate aus Tusculanae disputationes folgen: M. Tulli Ciceronis scripta quae manserunt omnia. Fasc. 44. Tusculanae disputationes. Hrsg. von M. POHLENZ. Stuttgart 1918. Zürich 1950.

${ }^{23}$ Alle Zitate aus De officiis folgen: M. T. Ciceronis De officiis libri III. Hrsg. von O. GIGON.

${ }^{24} \mathrm{Zu}$ den Tusculanen als Trost für den Autor vgl. Tusc. 5. 121: ... nostris quidem acerbissimis doloribus variisque et undique circumfusis molestiis alia nulla potuit inveniri levatio. 
gen des Verlusts seiner Tochter und gleichzeitig ist er von den aktuellen Ereignissen im Staat schwer betroffen.

Anfang September 44 v. Chr., nachdem Cicero seine erste Rede gegen Marc Anton im Senat gehalten hatte, war der Krieg zwischen ihnen schon Realität. Cicero verließ Rom und widmete sich in seinem Landhaus in Tusculum der Philosophie. Aus dieser Zeit stammt ein großer Teil seiner philosophischen Texte. ${ }^{25}$ In off. 2. 1.3 klagt Cicero darüber, dass er keinen Zugang mehr zu den öffentlichen Arbeiten hat. Wäre es ihm möglich, sich wieder dem Staat zu widmen, hätte er lieber über andere Themen geschrieben: Primum enim, ut stante re publica facere solebamus, in agendo plus quam in scribendo operae poneremus, deinde ipsis scriptis non ea, quae nunc, sed actiones nostras mandaremus, ut saepe fecimus. An dieser Stelle kann wiederum eine Übereinstimmung mit der augustinischen Beschreibung der zweiten Gruppe navigantes festgestellt werden. Das sind Menschen, die weinend und klagend durch einen Sturm im Hafen der Philosophie ankommen.

Laut der eigenen Aussage kommt Cicero zur Philosophie, um sich von den molestiae zu befreien: off. 2. 1. 4: existimavi honestissime molestiae posse deponi, si me ad philosophiam retulissem; off. 2. 2. 6: Nam sive oblectatio quaeritur animi requiesque curarum, quae conferri cum eorum studiis potest, qui semper aliquid anquirunt, quod spectet et valeat ad bene beateque vivendum? Seinem Unglück aber verdankt er das Glück, über Themen schreiben zu können, welche in Rom noch nicht bekannt waren: off. 2. 2. 5: Maximis igitur in malis hoc tamen boni assecuti videmur, ut ea litteris mandaremus, quae nec erant satis nota nostris... Diese Stelle korrespondiert mit der Bemerkung Augustins, dass die Menschen der zweiten Gruppe oft eine tempestas brauchen, um sich an die Philosophie zu wenden. Die molestiae und maxima mala können parallel zu altissima miseriarum (Z. 26) betrachtet werden. Wie bekannt, war der homo novus Cicero besonders ambitiös. Verlockt von der Möglichkeit honores zu bekommen, verlässt er die Philosophie, um sich der politischen Karriere zu widmen: off. 2. 1. 4: Cui cum multum adulescens discendi causa temporis tribuissem posteaquam honoribus inservire coepi meque totum rei publicae tradidi. Die von Augustin beschriebenen Menschen gelangen in tiefstes Elend, verlockt von dem trügerischen Glanz von Vergnügungen und Ehren (Z. 27 f.). Hier ist die nächste Übereinstimmung der beiden Beschreibungen zu sehen.

Wenn mit patria im augustinischen Text die philosophia gemeint ist, fällt schon die nächste Parallele ins Auge. Nachdem Cicero als Junge den Hafen der Philosophie verlassen hatte, ${ }^{26}$ kehrte er, nachdem in Rom Marc Anton an die Macht gekommen war, wieder zur Philosophie zurück (vgl. off. 2. 1. 4: Cui cum multum adulescens discendi causa temporis tribuissem; Tusc. 5. 5: Cuius (scil. philosophiae) in sinum cum a primis temporibus aetatis nostra voluntas studiumque nos compulisset, his gravissimis casibus in eundem portum, ex quo eramus egressi, magna iactati tempestate

${ }^{25}$ Vgl. off. 1. 1. 3 .

${ }^{26}$ Als Junge bemühte sich Cicero sehr um die Philosophie. Er war Schüler von Philon von Larisa, der letzte Scholarch der Platonischen Akademie, und vom Stoiker Diodotos. Später (79-78 v. Chr.) besuchte er Athen, wo er Antiochos von Askalon hörte, und Rhodos, wo er den Stoiker Posidonius kennenlernte. 
confugimus). Die Zitate beweisen, dass das Motiv für die Rückkehr (zur Philosophie) auch bei Cicero vorhanden ist: Dank einem heftigen Sturm kehrte er in den Hafen der Philosophie zurück. Ciceros Beschreibung der eigenen Rückkehr entspricht der Bemerkung Augustins, dass der zweiten Gruppe Seefahrer ein tobender Sturm zu wünschen ist, durch den sie in den Hafen kommen.

Mit den oben gezogenen Parallelen soll für den Vorschlag, dass in der zweiten Gruppe navigantes, die Augustin in beata v. 1. 2 beschreibt, Cicero gemeint sein kann, argumentiert werden. Diese Zuordnung kann durch die Annahme erklärt werden, dass Augustin in den ersten zwei Gruppen die Hinwendung zur Philosophie der beiden Philosophen Theodorus und Cicero, durch welche er für die Beschäftigung mit der Philosophie begeistert wurde ${ }^{27}$ schilderte, bevor er in der dritten Gruppe seinen eigenen Weg zur Philosophie beschrieb.

Zum Schluss müssen noch die beiden oben gestellten Fragen, warum die drei Textabschnitte, in welchen die drei Arten Seefahrer vorgestellt werden, unterschiedlich lang sind und warum Augustin selbst keine Beispiele für Repräsentanten jeder Gruppe nennt, beantwortet werden. Die beiden Fragen stehen in engem Zusammenhang und damit die erste Frage eine Antwort bekommt, muss zuerst die zweite Frage beantwortet werden. Obwohl Augustin mit der antiken Tradition der Illustrierung wichtiger Aussagen mit Beispielen vertraut war ${ }^{28}$ nannte er an dieser Stelle keine konkreten Personen, weil für die zeitgenössischen Leser des Dialogs klar sein sollte, wer in jeder Gruppe gemeint ist. Da die Repräsentanten der ersten und der dritten Gruppe (Theodorus bzw. Augustin) Zeitgenossen der Leser waren, war es nicht nötig, dass der Autor sie ausführlicher beschreibt. Deshalb ist der Text über sie wesentlich kürzer. Wenn angenommen wird, dass in der zweiten Gruppe Cicero gemeint ist, wird auch die Länge des Textes in diesem Teil verständlich. Auch der Leser im 4. Jhd. soll eine detailliertere Beschreibung gebraucht haben, um hinter dem Bild der Menschen in der zweiten Gruppe Cicero zu sehen.

Mit dem vorliegenden Text möchte die Autorin keinesfalls die im Moment in der Forschung akzeptierte Meinung bestreiten, dass es sich bei der zweiten Gruppe um Romanianus handelt, sondern den Vorschlag unterbreiten, dass es sich möglicherweise auch um Cicero handeln könnte. In einer Diskussion darüber könnte man sich über die eine oder die andere Person einigen, oder es könnten sogar andere Vorschläge gemacht werden.

\author{
Iglika Milusheva \\ Paris-Lodron-Universität Salzburg \\ Kultur- und Gesellschaftswissenschaftliche Fakultät \\ Fachbereich Altertumswissenschaften / Klassische Philologie \\ und Wirkungsgeschichte der Antike \\ iglika.milusheva@sbg.stud.ac.at
}

${ }^{27} \mathrm{Vgl}$. beata v. 1.4 75-78: Ego ab usque undevicesimo anno aetatis meae, postquam in schola rhetoris librum illum Ciceronis, qui Hortensius vocatur, accipi, tanto amore philosophiae succensus sum...; ibid. 98-100: Lectis autem Plotini paucissimis libris, cuius te (scil. Theodorum) esse studiosissimum accepi, ... sic exarsi...

${ }^{28}$ Dazu vgl. z. B. beata v. 4. 26, wo Augustin auf das Beispiel des Orata zurückgreift. 